\title{
The Surface Proximity Effect on the Formation of Extended Defects in Ion Beam Synthesised SiGe/Si Heterostructures
}

\author{
F. Cristiano, A. Nejim and P.L.F. Hemment \\ School of Electronic \& Electrical Engineering, Information Technology and Mathematics \\ University of Surrey \\ Guildford, Surrey GU2 5XH, UK
}

\begin{abstract}
The relaxation of ion beam synthesised SiGe alloys occurs during solid phase epitaxial growth (SPEG) by a roughening of the amorphous/crystalline $(\mathrm{a} / \mathrm{c})$ interface, leading to the formation of extended defects. SiGe/Si structures have been formed by implantation of $\mathrm{Ge}^{+}$ions at energies of $70 \mathrm{keV}$ and $400 \mathrm{keV}$ and doses above the critical value for strain relaxation, followed by post-amorphisation to a depth of $\sim 1 \mu \mathrm{m}$ and regrowth at $700^{\circ} \mathrm{C}$. TEM and RBS analysis of the regrown structures show that relaxation-induced stacking faults (SFs) are nucleated in the vicinity of the peak of the Ge concentration. For an implantation energy of $70 \mathrm{keV}$, SFs are the only defects observed and they extend up to the surface. For an implantation energy of 400 $\mathrm{keV}$, the SFs terminate within the bulk far from the surface, while "hairpin" dislocations are also formed and extend up to the surface. These results are explained in terms of the ratio between the depth at which SFs are nucleated and the roughness of the $\mathrm{a} / \mathrm{c}$ interface at that depth. Varying the defect nucleation depth by removing a surface layer from the structures implanted at $400 \mathrm{keV}$ prior to SPEG does not result in a change of the size of the SFs, while "hairpin" dislocations are still formed, suggesting that the roughness of the a/c interface during regrowth is only determined by the $\mathrm{Ge}$ content in the alloy.
\end{abstract}

\section{INTRODUCTION}

Ion implantation has been proposed as one of the possible technologies to produce device worthy $\mathrm{SiGe} / \mathrm{Si}$ heterostructures [1], [2]. As a consequence of ion implantation, the near surface layer is amorphised, calling for a solid phase epitaxial growth (SPEG) stage during which the accumulation of elastic strain may result in the formation of extended defects. It has been shown by Paine et al [3], [4], that the introduction of strain relieving stacking faults (SFs) is facilitated by a change from a planar (001) growth surface to one which is faceted parallel to the crystal $\{111\}$ planes. This description has been confirmed by Elliman et al [5] who used time resolved reflectivity (TRR) measurements to show that the roughness $(r(z))$ of the amorphous/crystalline $(\mathrm{a} / \mathrm{c})$ interface at a particular depth $(z)$ during regrowth is directely proportional to the germanium concentration at that depth. However, although in the work of Paine the authors reported the presence of SFs nucleated in the vicinity of the peak of the $\mathrm{Ge}$ depth profile, no SFs were found to extend up to the surface in fully regrown structures synthesised by implantation of $200 \mathrm{keV} \mathrm{Ge}^{+}$ions. Instead, long threadiri.g dislocations were observed, similar to the "hairpin" dislocations previously reported by our group [6], [1] in structures synthesised by implantation of $400 \mathrm{keV} \mathrm{Ge}^{+}$ions. On the other hand, relaxation-induced SFs have been found to extend to the surface with no "hairpin" dislocation formation in structures formed using lower $\mathrm{Ge}^{+}$implantation energy (120 keV in [7] or $160 \mathrm{keV}$ in [8]).

The aim of this experiment is to identify which type of relaxation-induced extended defects is formed after regrowth of SiGe alloy layers synthesised using different values of the $\mathrm{Ge}^{+}$implantation energy. In addition, the influence of the surface proximity on the type of defect formed is also investigated. The TEM and RBS results are discussed in terms of the ratio between the depth at which the relaxation-induced defects are nucleated and the roughness of the $\mathrm{a} / \mathrm{c}$ interface at that depth.

\section{ExPERIMENTAL DETAILS}

3 " p-type (10 $\Omega$-cm) (100) $\mathrm{Cz}$ silicon wafers were implanted with $\mathrm{Ge}^{+}$ions at energies of $70 \mathrm{keV}$ and $400 \mathrm{keV}$ to doses of $4 \times 10^{16} \mathrm{Ge}^{+} / \mathrm{cm}^{2}$ and $1 \times 10^{17} \mathrm{Ge}^{+} / \mathrm{cm}^{2}$, respectively. Both $\mathrm{Ge}^{+}$doses are above the critical value for strain relaxation for the implantation energy used in this study [9]. The wafers were subsequently post amorphised down to a depth of $\sim 1 \mu \mathrm{m}$ using $500 \mathrm{keV} 6 \times 10^{15} \mathrm{Si}^{+} / \mathrm{cm}^{2}$ implanted at $150 \mathrm{~K}$ using liquid nitrogen. Selected samples from wafer 2 (see table I) were thinned using anodic oxidation in a 0.05 molar solution of $\mathrm{KNO}_{3}$ in $90 \%$ ethylene Glycol and $10 \%$ de-ionised water. This solution produced anodisation coefficient of $0.25 \mathrm{~nm} / \mathrm{V}$. Using platinum wire as the cathode and the samples as the anode a constant current supply of $90 \mathrm{~mA}$ and $100 \mathrm{~V}$ were used to oxidise $25 \mathrm{~nm}$ per run. This oxide was sybsequently removed in HF solution. The process was repeated to remove layers of different thickness. All samples were finally annealed at $700^{\circ} \mathrm{C}$ for $20 \mathrm{~min}$ in flowing $\mathrm{N}_{2}$ in an RTA unit. The various process steps are also schematically shown in figure 1.

The thickness of the remaining amorphous layers in the various samples prior to SPEG was measured by RBS. The corresponding values of the thickness removed by anodic oxidation are reported in table I. Due to non-uniformity in the efficiency of the anodisation process across the samples, surface roughening was observed after regrowth of the oxidised samples. However, the roughness values were found to be low even after several anodisation runs. After removing a surface layer of almost $200 \mathrm{~nm}$ the average value of the surface roughness was measured to be about $9 \mathrm{~nm}$, with a maximum "peak-to-valley" distance of about $15 \mathrm{~nm}$.

In addition to the thickness of the amorphous layers, the germanium doses and the germanium depth distribution parameters shown in table I were also determined by RBS 
analysis, performed using $1.5 \mathrm{MeV} \mathrm{He}{ }^{+}$ions with an overall system resolution of $14 \mathrm{keV}$. The microstructure of the synthesised structures after regrowth was investigated by plan view and cross sectional TEM using either a JEOL 2000-FX or a Philips 400T electron microscope.

\section{RESULTS AND DISCUSSION}

Figure 2(a) shows a XTEM micrograph from sample 1 where a layer of crystallographic defects is observed extending from a depth of about $50 \mathrm{~nm}$ up to the surface. The extended defects include "V"-shaped stacking faults lying on $\{111\}$ planes, with a density of the order of $1 \times 10^{10} / \mathrm{cm}^{2}$. The peak germanium concentration for this sample is 11.4 at.\%. A lower magnification image (not shown) also shows a layer of EOR defects at a depth of $1 \mu \mathrm{m}$, corresponding to the thickness of the amorphous layer formed during the post-amorphisation step. Assuming the simple relation given by Elliman [5] between the roughness of the (a/c) interface at a particular depth during regrowth and the germanium concentration at that depth, it is found for sample 1 , implanted with $4 \times 10^{16} \mathrm{Ge}^{+} / \mathrm{cm}^{2}$ at $70 \mathrm{keV}$, that strain relieving stacking faults were formed at a depth of $50 \mathrm{~nm}$, which is comparable to the estimated value of the roughness of the a/c interface $(30 \mathrm{~nm})$. This situation is schematically described in figure $2(\mathrm{~b})$, where it is shown that the faceting of the $a / c$ interface, responsible for the formation of strain relieving SFs, occurs at a depth close to the surface. Under such contidions, SFs are easily terminated at the free surface, storing all the elastic energy released in the process.

Figure 3(a) shows a XTEM micrograph from sample 2, implanted with $1 \times 10^{17} \mathrm{Ge}^{+} / \mathrm{cm}^{2}$ at $400 \mathrm{keV}$. "Hairpin" dislocations are observed in the surface region of the sample. They are formed at a depth of about $0.25 \mu \mathrm{m}$ and extend up to the surface. This depth corresponds to the $\bar{R}_{p}$ of the implanted $\mathrm{Ge}^{+}$ions where the Ge concentration has a maximum value of 7.8 at.\%. The density of "hairpin" dislocations, determined by plan view TEM (not shown), has been found to be $(1.5 \pm 0.35) \times 10^{9} \mathrm{disl} / \mathrm{cm}^{2}$. Similar to the previous sample, a layer of EOR defects is observed at a depth of $1 \mu \mathrm{m}$. However, a high magnification XTEM image from the same sample (figure 5(a)), taken from a region close to the $\overline{R_{p}}$ of the germanium distribution profile, shows that SFs are also formed in this structure, with an average size of $30 \mathrm{~nm}$, which are terminated within the bulk. No SFs were observed either above or below the " $\overline{R_{p}} "$ region. Using the same argument employed for the low energy implanted sample, it is found for sample 2 that strain relieving SFs and "hairpin" dislocations were formed at a depth of $\sim 250 \mathrm{~nm}$, where the estimated value of the roughness of the a/c interface is $20 \mathrm{~nm}$, which is more than ten times smaller. This situation is schematically described in figure 4, where it is shown that the faceting of the a/c interface, responsible for the formation of strain relieving SFs, occurs at a depth far away from the surface. Under such contidions, SFs cannot extend up to the surface, as the energy required would exceed the available elastic energy associated with the SiGe alloy layer [10]. In addition, as the moving a/c interface passes through the peak of the germanium depth profile, crystallization proceeds in a region of decreasing germanium concentration, leading to a smoothing of the a/c interface [5]. Therefore the probability of new SFs to be nucleated close to the surface is low in agreement with the TEM results.

The effect of the proximity of the free surface on the formation of relaxation-induced defects in samples implanted with $400 \mathrm{keV} \mathrm{Ge}{ }^{+}$ions is described in figure $3 \mathrm{~b}, \mathrm{c}$ and $\mathrm{d}$ where XTEM micrographs from samples $2 \mathrm{~b}, 2 \mathrm{c}$ and $2 \mathrm{~d}$ are presented, respectively. The layer of EOR defects associated with the $\mathrm{Si}^{+}$post-amorphisation step is observed in all the samples, at a depth depending on the thickness of the surface layer removed by anodic oxidation and $\mathrm{HF}$ etching (see table I). For a removed layer considerably thinner than $\overline{R_{p}}$ (samples $2 \mathrm{~b}, 2 \mathrm{c}$ in figure $3 \mathrm{~b}$ and $\mathrm{c}$, respectively), "hairpin" dislocations are still observed, whose length is consistent with the thickness of the removed layer. On the other hand "hairpin" dislocations are no longer observed in sample 2d (figure 3d) where a surface layer of $180 \mathrm{~nm}$ was removed before SPEG, comparable to the depth corresponding to the $\overline{R_{p}}$ of the implanted $\mathrm{Ge}^{+}$ions. High magnification XTEM micrographs from the same samples are shown in figure $5 \mathrm{~b}, \mathrm{c}$ and $\mathrm{d}$, respectively. It is observed that SFs are still formed both within the bulk as well as on the surface of the samples. However, their size never exceeds $30 \mathrm{~nm}$, as in the reference non-etched sample (figire 5a). These results suggest that, upon removal of a thin surface layer, the ratio between the defect nucleation depth and the a/c interface roughness decreases, allowing some of the SFs to extend up to the surface. However, the size of the SFs is not found to increase, indicating that the a/c interface roughness is not affected by the proximity of the free surface but it is determined by the Ge content only.

\section{Conclusions}

$\mathrm{SiGe} / \mathrm{Si}$ structures have been formed by implantation of $\mathrm{Ge}^{+}$ions at energies of $70 \mathrm{keV}$ and $400 \mathrm{keV}$ and doses above the critical value for strain relaxation, followed by post-amorphisation to a depth of $\sim 1 \mu \mathrm{m}$ and regrowth at $700^{\circ} \mathrm{C}$. Surface layers of different thicknesses were removed from selected samples before SPEG using anodic oxidation and HF etching. From the TEM and RBS analysis it is concluded that:

- The extended defects formed upon relaxation of the strain associated with a $\mathrm{SiGe/Si}$ heterostructure synthesised by $\mathrm{Ge}^{+}$implantation consist of SFs nucleated in the vicinity of the peak of the germanium concentration.

- The relaxation induced SFs extend up to the surface for a low $\mathrm{Ge}^{+}$implantation energy $(70 \mathrm{keV})$ or terminate within the bulk for a higher $\mathrm{Ge}^{+}$implantation energy ( $400 \mathrm{keV})$ depending on the ratio between the depth at which the defects are nucleated and the roughness of the a/c interface at that depth during SPEG. For a high Ge ${ }^{+}$implantation energy, "hairpin" dislocations which extend up to the surface are also formed together with the SFs. 
- The size of the SFs is not affected by the proximity of the free surface to the a/c interface during SPEG, while "hairpin" dislocations are no longer observed when the thickness of the removed layer is close to the defect nucleation depth.

\section{ACKNOWLEDGMENTS}

The authors wish to acknowledge Chris Jeynes, from the University of Surrey, for the RBS measurements of the etched samples before regrowth.

\section{REFERENCES}

[1] F. Cristiano, A. Nejim, B. de Mauduit, A. Claverie, and P.L.F. Hemment, "Characterization of extended defects in SiGe alloys formed by high dose $\mathrm{Ge}^{+}$implantation into $\mathrm{Si}$," Nucl. Instr, and Meth., vol. B120, pp. 156-160, 1996.

[2] S. Lombardo, A. Pinto, V. Raineri, P. Ward, G. La Rosa, G. Privitera, and S.U. Campisano, " $\mathrm{Si} / \mathbf{G e}_{x} \mathbf{S i}_{1-x}$ heterojunction bipolar transistors with the $\mathrm{Ge}_{x} \mathrm{Si}_{1-x}$ base formed by Ge ion implantation in Si," IEEE Elec. Dev. Lett., vol. 17 , no. 10, pp. 485-487, October 1996.

[3] D.C. Paine, D.J. Howard, N.G. Stoffel, and J.A. Horton, "The growth of strained $\mathrm{Si}_{1-x} \mathrm{Ge}_{x}$ alloys on $<001>$ silicon using solid phase epitaxy," J. Mater. Res., vol. 5(5), pp. 10231031,1990 .

[4] D.C. Paine, D.J. Howard, and N.G. Stoffel, "Strain relief in compositionally graded $\mathrm{Si}_{1-x} \mathbf{G e}_{x}$ formed by high dose ion implantation," J. Electronic Mater., vol. 20(10), pp. 735$746,1991$.

[5] R.G. Elliman and W.C. Wong, "Kinetic roughening and smoothing of the crystalline-amorphous interface during solid phase epitaxial crystallization of GeSi alloy layers," Appl. Phys. Lett., vol. 69, no. 18, pp. 2677-2679, 1996.

[6] F. Cristiano, J.P. Zhang, R.J. Wilson, W.P. Gillin, and P.L.F. Hemment, "Control of defects in $\mathrm{C}^{+}, \mathrm{Ge}^{+}$and $\mathrm{Er}^{+}$implanted Si using post amorphization and solid phase regrowth," Nucl. Instr, and Meth., vol. B96, pp. 265-270, 1995.

[7] S. Im, J. Washburn, R. Gronsky, N.W. Cheung, and K.M. Yu, "Defect control during solid phase epitaxial growth of SiGe alloy layers.," Appl. Phys. Lett., vol. 63, no. 7, pp. 929$931,1993$.

[8] A. Fukami, K. Shoji, T. Nagano, T. Tokuyama, and C.Y. Yang, "Graded-bandgap SiGe bipolar Transistor fabricated with germanium ion implantation," Microelectr. Engineering, vol. 15, pp. 15-18, 1991.

[9] F. Cristiano, A. Nejim, Yu. Suprun-Belevich, A. Claverie, and P.L.F. Hemment, "Formation of extended defects and strain relaxation in ion beam synthesised SiGe alloys," To be published in Nucl. Instr, and Meth. B, E-MRS 1998.

[10] F. Cristiano, Extended defects in SiGe device structures formed by ion implantation, Ph.D. thesis, University of Surrey, Guildford, UK, March 1998.

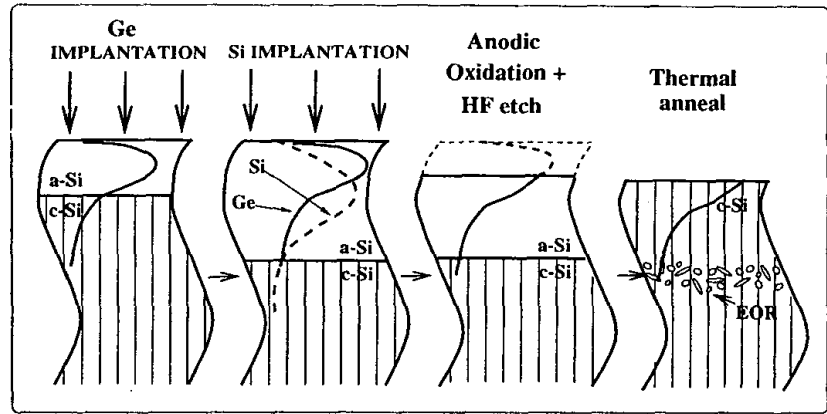

Fig. 1. Schematic diagram of the processing steps for the formation of SiGe/Si structures by ion implantation and anodic oxidation.

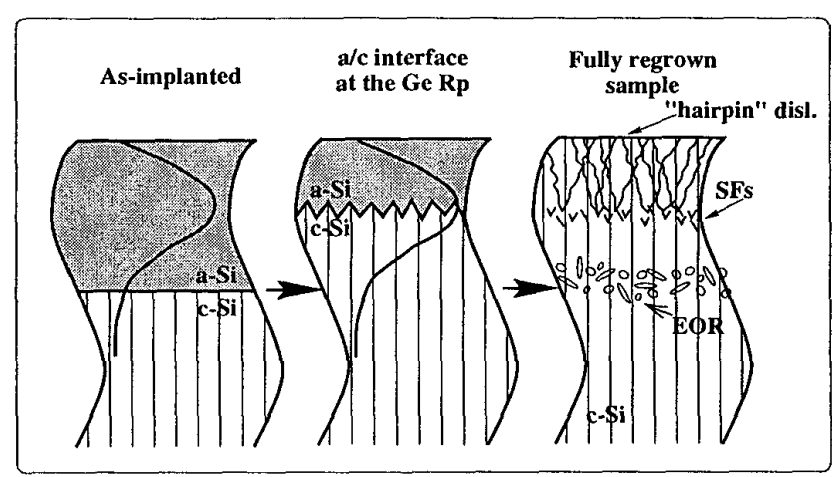

Fig. 4. Schematic diagram illustrating the roughening of the $\mathrm{a} / \mathrm{c}$ interface and the formation of SFs terminating within the bulk and "hairpin" dislocation extending up to the surface during SPEG of SiGe alloys synthesised at an implantation energy of $400 \mathrm{keV}$.

TABLE I

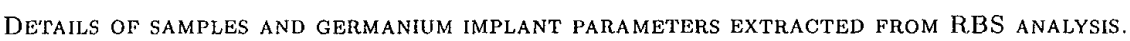

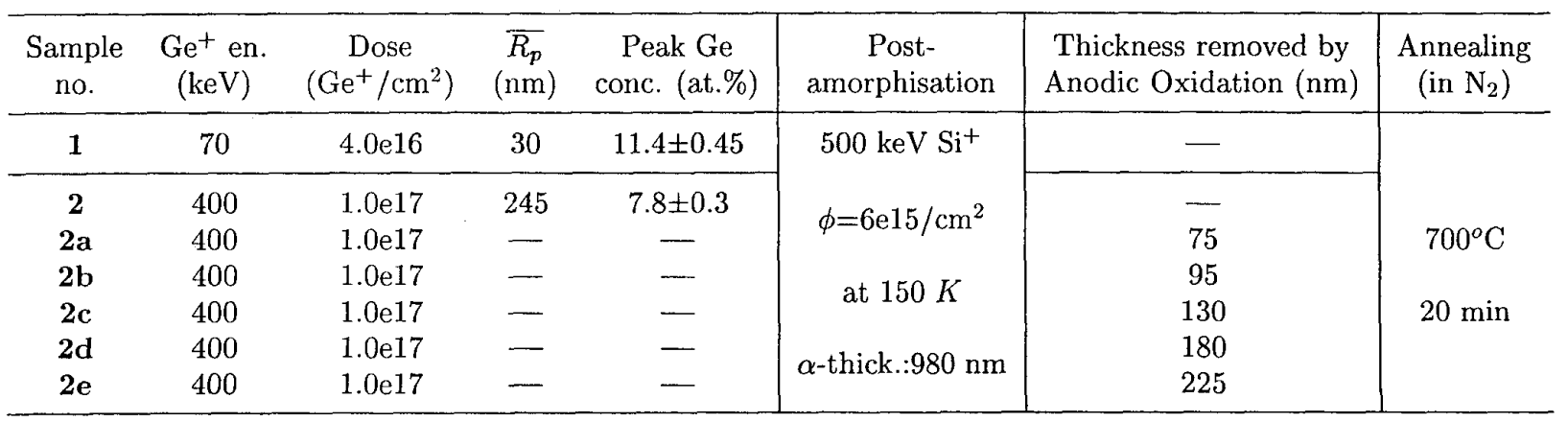




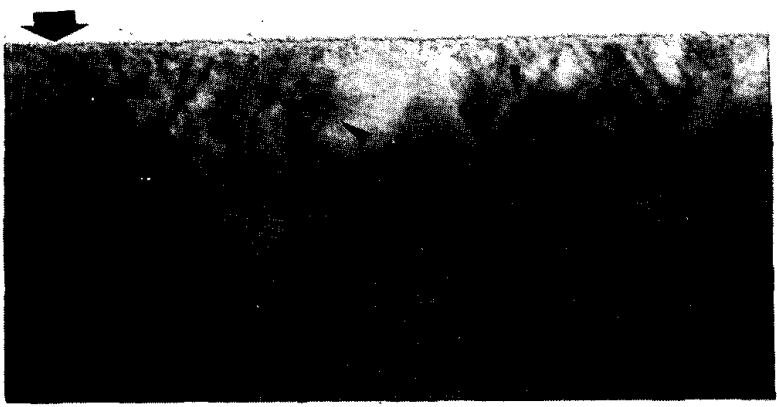

(a)

\section{$100 \mathrm{~nm}$}

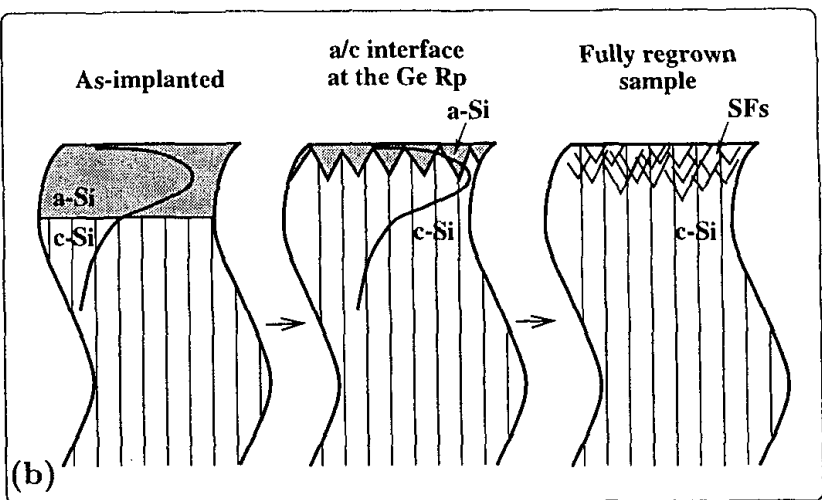

(b)

Fig. 2. (a) XTEM bright field micrograph from sample 1, implanted with $70 \mathrm{keV} \mathrm{Ge}+$ ions, after post-amorphisation and regrowth at $700^{\circ} \mathrm{C}$ for $20 \mathrm{~min}$. (b) Schematic diagram illustrating the roughening of the a/c interface and the formation of SFs terminating at the surface during SPEG of SiGe alloys synthesised at an implantation energy of $70 \mathrm{keV}$.

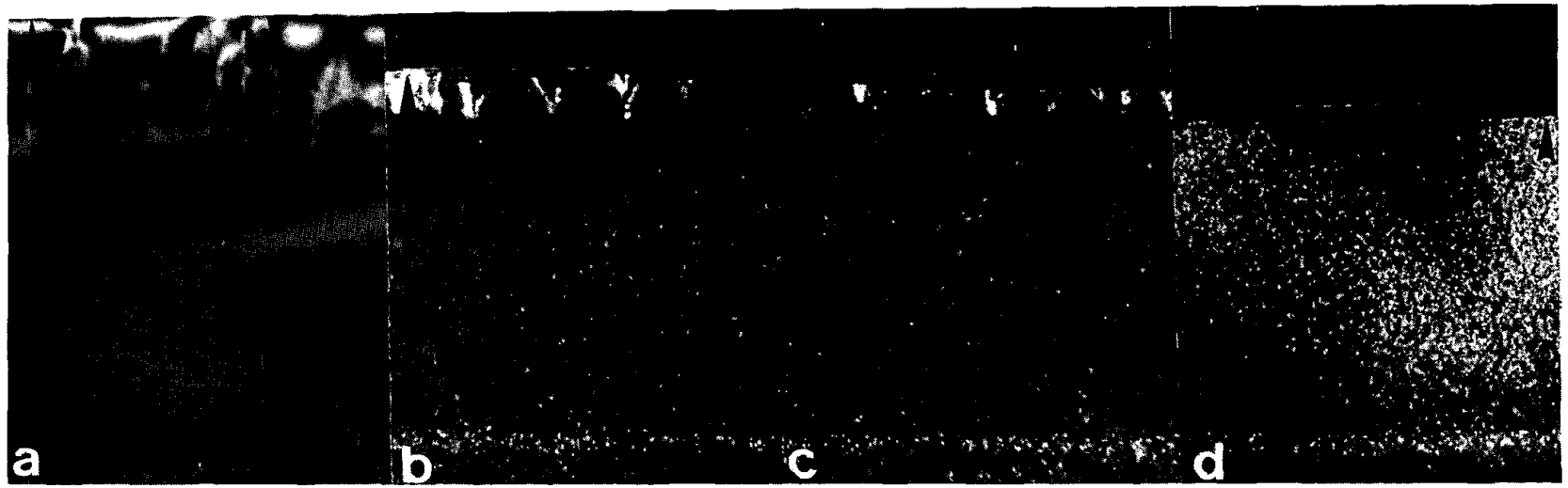

\section{$500 \mathrm{~nm}$}

Fig. 3. XTEM micrographs from samples implanted with $400 \mathrm{keV} \mathrm{Ge}+$ ions after removal of different surface layers and regrowth at $700^{\circ} \mathrm{C}$ (a) Sample 2 (bright field). (b) Sample 2b (weak beam dark field). (c) Sample 2c (weak beam dark field). (d) Sample 2d (weak beam dark field).

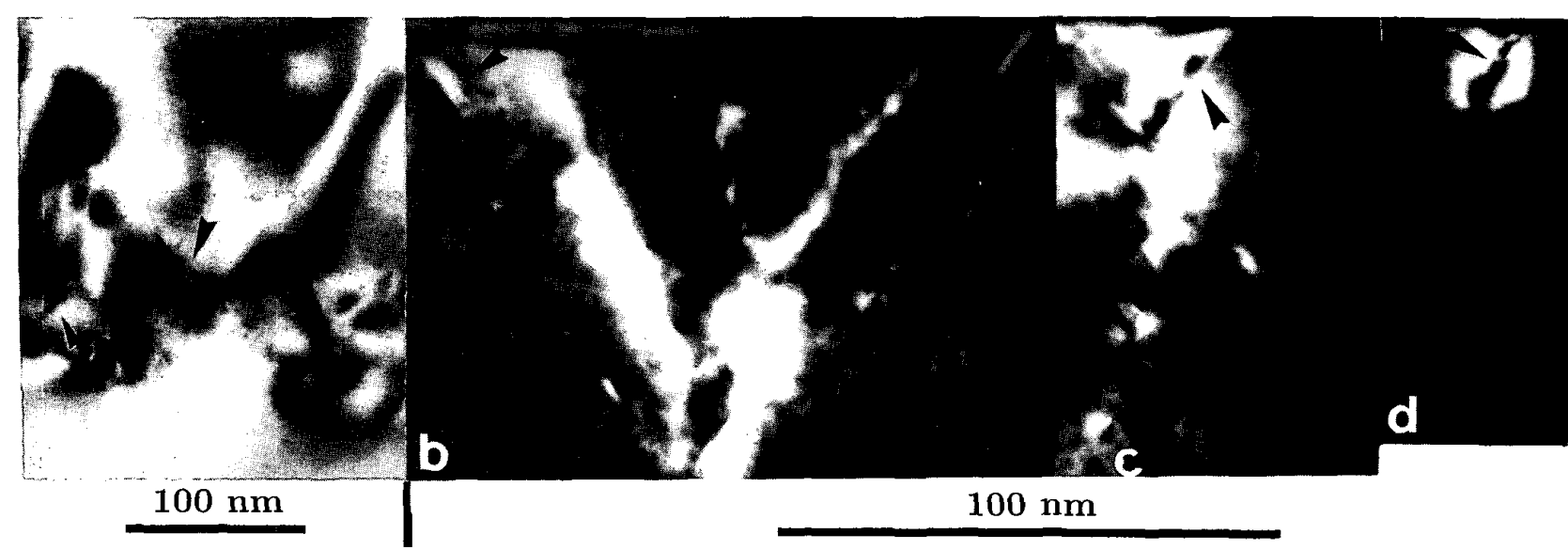

Fig. 5. High magnification XTEM micrographs from samples implanted with $400 \mathrm{keV} \mathrm{Ge}+$ ions (see figure 3) after removal of different surface layers and regrowth at $700^{\circ} \mathrm{C}$. (a) Sample 2 (bright field). (b) Sample $2 \mathrm{~b}$ (dark field). (c) Sample 2c (dark field). (d) Sample 2d
(dark field). 